\title{
TEORES DE CARBAMATO DE ETILA EM AGUARDENTES DE CANA E MANDIOCA. PARTE II
}

\author{
Luiz Gualberto de Andrade Sobrinho, Luciana Tereza Dias Cappelini, Alexandre Ataíde da Silva, Carlos Alexandre \\ Galinaro, Silmara França Buchviser, Daniel Rodrigues Cardoso e Douglas Wagner Franco*
}

Instituto de Química de São Carlos, Universidade de São Paulo, CP 780, 13560-970 São Carlos - SP, Brasil

Recebido em 13/2/08; aceito em 14/8/08; publicado na web em 18/12/08

CONTENTS OF ETHYL CARBAMATE IN SUGAR CANE AND MANIOC SPIRITS. PART II. Herein, we report the concentration of ethyl carbamate (EC) and copper in 380 samples of sugar-cane spirit and 45 samples of manioc spirit as determined by GC-MS and FAAS respectively. The cyanide content determined spectrophotometrically is reported for the manioc spirit. Sugar cane spirit produced by alembic distillation $\left(70,0 \mu \mathrm{L} \mathrm{L}^{-1}\right)$ shown a lower content of EC than samples produced by column distillation $(270 \mu \mathrm{g}$ $\mathrm{L}^{-1}$ ). No simple correlation between the content of EC and copper for sugar cane spirit as well among the concentration of EC, copper, and cyanide for manioc spirit could be observed.

Keywords: sugar-cane spirit; manioc spirit; ethyl carbamate.

\section{INTRODUÇÃO}

O carbamato de etila (CE), substância com reconhecido potencial carcinogênico, ${ }^{1}$ juntamente com os hidrocarbonetos policíclicos aromáticos (HPAs) ${ }^{2}$ e os flocos de dextranas, ${ }^{3}$ estão entre os principais "defeitos da aguardente".

Apesar de constantes alertas do Prof. F. V. Novaes (ESALQ) e da comunidade científica (FEA/UNICAMP e IQSC/USP), somente em 2005, o governo brasileiro através do Ministério da Agricultura, Pecuária e Abastecimento (MAPA), ${ }^{4}$ estabeleceu o limite máximo de CE para a aguardente de cana em $150 \mu \mathrm{L} \mathrm{L}^{-1}$ (150 ppb).

Em vinhos, a concentração de CE formada durante e depois da fermentação é proveniente da reação do etanol com os precursores do carbamato, tais como, uréia, citrulina e dietil pirocarbonato. ${ }^{5,6} \mathrm{~A}$ concentração de etanol e dos precursores, bem como a temperatura, o tempo de estocagem e a luz são importantes parâmetros para formação do $\mathrm{CE}$ em vinhos, ${ }^{7}$ o qual geralmente apresenta seus teores variando de 10 a $50 \mu \mathrm{g} \mathrm{L} \mathrm{L}^{-1}$.

As reações responsáveis pela formação do CE são influenciadas pelo tipo de levedura, ${ }^{8,9}$ nutrientes adicionados ao mosto, ${ }^{8,9}$ concentração de etanol (teor alcoólico), ${ }^{10,11}$ temperatura de fermentação e acidez durante a fermentação. ${ }^{1,11} \mathrm{O}$ primeiro composto considerado como sendo o responsável pela formação do CE no vinho foi o dietil pirocarbonato, agente antimicrobiano utilizado no tratamento do mosto. ${ }^{12}$

A uréia é um dos principais precursores ${ }^{7,10}$ para a formação do CE em vinhos. A presença de uréia decorre da degradação da arginina, que é o principal aminoácido existente na uva.

Nos uísques recém destilados são os precursores voláteis, tais como cianeto, cianato, cianoidrina, tiocianato e o complexo cobrecianeto que induzem a formação de CE. ${ }^{1,13}$ A presença do cianeto no uísque escocês é proveniente da matéria-prima empregada, pois na cevada é encontrado o glicosídeo cianogênio, epieterodendrina, que por hidrólise enzimatimática libera o ácido ciânídrico $(\mathrm{HCN})$ e o isobutiraldeído. ${ }^{14} \mathrm{~A}$ identificação desses precursores deve-se ao fato de que a concentração do CE aumenta ao mesmo tempo em que ocorre a diminuição dos mesmos. ${ }^{14-18}$

A gênese do CE e de seus precursores em aguardente de cana e de mandioca, tiquira, vem sendo estudada no Laboratório para o

\footnotetext{
*e-mail: douglas@iqsc.usp.br
}

Desenvolvimento da Química da Aguardente (LDQA). ${ }^{19-22}$ Como parte destes esforços, neste trabalho são relatados os resultados sobre a influência do tipo de destilação, do tempo de estocagem, da coloração do vasilhame e da presença de íons de cobre e cianeto nas concentrações de $\mathrm{CE}$ em aguardentes.

Uma avaliação da variação do teor de CE em amostras de aguardentes, produzidas ao longo dos últimos anos também é apresentada.

\section{PARTE EXPERIMENTAL}

Foram analisadas amostras comerciais de aguardente produzidas entre 2001 a 2006, perfazendo um total de 380 amostras. Dentre essas, 202 amostras foram adquiridas no comércio, 70 participaram dos V e VI Brazilian Meeting on Chemistry of Food and Beverages (V e VI $\mathrm{BMCFB})$ e 108 foram coletadas na origem, como parte do Projeto de Tipificação da Aguardente do Estado de São Paulo (FAPESP processo $n^{\mathbf{0}}$ 2001/12934). Para as amostras de aguardente de cana, determinaram-se as concentrações de $\mathrm{CE}$ e de íons de cobre.

Também foram coletadas e analisadas 45 amostras de aguardente de mandioca (tiquira), provenientes de diferentes cidades do estado do Maranhão. Nas amostras de tiquira, foram determinadas as concentrações de $\mathrm{CE}$, íons de cobre e cianeto.

Para a determinação das concentrações de CE, utilizou-se um cromatógrafo a gás Shimadzu 17A, acoplado a um detector seletivo de massas Shimadzu QP-5050A. A separação cromatográfica foi efetuada em uma coluna capilar de fase polar, HP-FFAP, com filme de polietilenoglicol esterificado, seguindo metodologia descrita anteriormente. ${ }^{19}$ Os limites de detecção (LD) e quantificação (LQ) para o CE foram de 10 e $40 \mu \mathrm{g} \mathrm{L}{ }^{-1}$, respectivamente.

A concentração de íons de cobre foi determinada utilizando-se um espectrofotômetro de absorção atômica com ionização por chama, ${ }^{23}$ Hitachi modelo Z-8100.

Para a determinação de cianeto, seguiu-se o método colorimétrico proposto por Lambert et al. ${ }^{24}$ Neste procedimento o íon cianeto é oxidado por uma solução de clorosuccinamida/succinimida e, em seguida, a solução resultante é adicionada a uma solução contendo ácido barbitúrico/piridina. O produto final da reação foi monitorado em 580 nm, utilizando-se um espectrofotômetro UV-visível Hitachi modelo U-3501. 


\section{RESULTADOS E DISCUSSÃO}

\section{Influência do tempo de estocagem na concentração do carbamato de etila}

Da produção da aguardente de cana ao seu engarrafamento pode transcorrer um período de até 6 meses, conhecido como processo de “descanso". A esse período, deve-se ainda acrescentar o tempo médio de permanência em prateleira, o qual é estimado em 6 meses. Desta forma, para verificar se há alteração da concentração de CE com o tempo, reanalisou-se a concentração desse composto em 15 amostras de aguardente de cana após 36 meses de repouso e ao abrigo da luz (Material Suplementar, Tabela 1S). Observou-se que as diferenças entre as concentrações de CE encontradas para estas amostras apresentaram uma variação de $-5,3$ a $6,7 \%$, sugerindo que este composto é estável após a sua formação.

\section{Influência do sistema de destilação na concentração do carbamato de etila}

Dois tipos de sistemas são utilizados para a destilação do vinho: alambique e coluna.

Os alambiques operam em modo descontínuo aquecidos a fogo direto ou a vapor. Podem ter de um a três corpos construídos de cobre ou aço inoxidável (cada corpo é equivalente a um prato teórico). São utilizados principalmente por pequenos produtores (produção inferior a $100.000 \mathrm{~L}$ por ano $)^{25}$ e o seu volume pode variar desde uma dezena até $1000 \mathrm{~L}$.

Nas colunas construídas em aço inoxidável, operando sob fluxo contínuo, o cobre encontra-se praticamente restrito às tubulações dos condensadores. As colunas apresentam múltiplos pratos teóricos originados por bandejas distribuídas em função da sua altura e diâmetro.

A Figura 1 apresenta o perfil da concentração de CE para um conjunto de 108 amostras de aguardente de cana coletadas no estado de São Paulo. Nota-se, claramente, que as amostras destiladas em alambique apresentam em sua maioria concentrações de $\mathrm{CE}$ entre $<$ LQ a $150 \mu \mathrm{g} \mathrm{L}^{-1}$. Para amostras destiladas em coluna esse ponto de acumulação situa-se na faixa de 151 a $400 \mu \mathrm{g} \mathrm{L}^{-1}$.

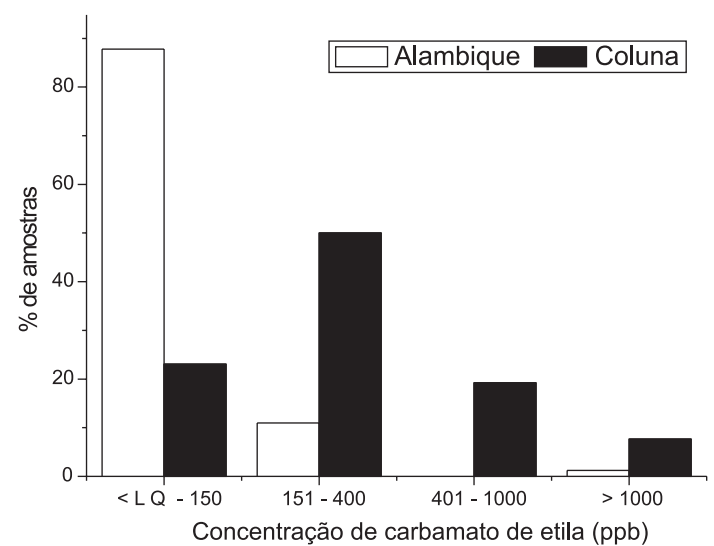

Figura 1. Distribuição de carbamato de etila em $\mu g L^{-1}$ (ppb), por faixas de concentração, em amostras de aguardente de cana destiladas em alambique e em coluna

Embora com perfis diferentes para a faixa de concentração entre 151 a $1,00 \times 10^{3} \mu \mathrm{g} \mathrm{L}^{-1}$, os resultados indicam de forma mais consistente a mesma tendência observada anteriormente ${ }^{19}$ para amostras de aguardente de cana comerciais oriundas das diversas regiões produtoras do país.
Em trabalho correlato, Bruno et al. ${ }^{26}$ determinaram as concentrações de CE em 34 amostras de aguardente de cana, coletadas em diferentes regiões do estado do Rio de Janeiro, produzidas utilizandose diferentes sistemas de destilação. Estes resultados também sugerem que os teores médios de CE presentes em aguardentes destiladas em alambiques são inferiores aos teores médios de aguardentes destiladas em coluna. ${ }^{26}$

É interessante ressaltar que na destilação em alambique são separadas três frações de destilado denominadas: cabeça, cauda e coração. O momento em que esta separação se efetua é um tanto arbitrário, mas geralmente os produtores utilizam para tal o grau alcoólico do destilado. Existem produtores que operam coletando a cabeça quando o grau alcoólico atinge o intervalo de 70 a $50 \%$ $\mathrm{v} / \mathrm{v},{ }^{26,27}$ o coração entre 50 a $38 \% \mathrm{v} / \mathrm{v}^{26-28}$ e a cauda entre 38 a $10 \%$ $\mathrm{v} / \mathrm{v}^{26,27} \mathrm{~A}$ aguardente de alambique é constituída basicamente da fração denominada coração.

Foram determinadas em nosso Laboratório as concentrações de CE nas três frações, cabeça, coração e cauda para 13 amostras de aguardente de cana destiladas em alambique e por nós coletadas na origem e no momento da sua destilação. Os valores das medianas referentes aos teores de $\mathrm{CE}$ que foram observados para cabeça, coração e cauda são, respectivamente, 130, 110 e 73,1 $\mu \mathrm{g} \mathrm{L}^{-1}$. Esses valores de concentração foram arbitrariamente corrigidos para um valor de graduação alcoólica de $38 \%$ v/v, devido este ser o teor alcoólico mais freqüentemente encontrado em aguardentes de cana comerciais.

A solubilidade do CE é maior em etanol $\left(1,2 \mathrm{~g} \mathrm{~mL}^{-1}\right)$ que em água $\left(0,10 \mathrm{~g} \mathrm{~mL}^{-1}\right) .{ }^{29}$ Assim é previsível que a fração cabeça, onde a concentração alcoólica é maior, apresente maiores concentrações de $\mathrm{CE}$ que as frações coração e cauda. Existindo um corte no produto artesanal, o que não ocorre no produto industrial, isto implicaria na redução do teor de $\mathrm{CE}$ do destilado.

Com a finalidade de melhor entender esta questão, foram analisadas amostras em que o mesmo vinho foi dividido em duas frações. Uma delas foi destilada em alambique e a outra em coluna, e então determinadas as concentrações de CE nos correspondentes destilados. A Tabela 1 apresenta resultados para amostras em que o mesmo vinho foi destilado em alambique e em coluna.

Tabela 1. Teores de carbamato de etila (CE) em amostras de aguardente de cana destiladas em alambique e coluna $\left(\mu \mathrm{g} \mathrm{L}^{-1}\right)$

Tipo de destilação

\begin{tabular}{lcc} 
Amostra* $^{*}$ & Alambique & Coluna \\
\hline 1 & 58,0 & 125 \\
2 & 216 & 644 \\
3 & 152 & 812 \\
\hline
\end{tabular}

* Cada amostra representa um diferente vinho fermentado, submetido à destilação em alambique de cobre e em coluna de aço inox

Os resultados apresentados na Tabela 1 indicam que as aguardentes de cana artesanais (alambique) tendem a apresentar teores de CE inferiores aos das aguardentes industriais (coluna). Esse fato não deve depender apenas do sistema de destilação, mas também da natureza do material utilizado na confecção destes aparelhos e dos diferentes procedimentos.

Para as mesmas 108 amostras de aguardente de cana anteriormente citadas, também foi realizada a análise de cobre, que apresentou um valor de mediana de concentração de $0,302 \mathrm{mg} \mathrm{L}^{-1}$, com valores mínimo e máximo de $1,00 \times 10^{-2}$ e $3,11 \mathrm{mg} \mathrm{L}^{-1}$, respectivamente (Material Suplementar, Tabela 2S).

Foram analisados também os teores de cobre em 45 amostras de tiquira (Material Suplementar, Tabela 3S). Observou-se que 51,1\% 
das amostras apresentam concentração deste íon acima de $5,0 \mathrm{mg} \mathrm{L}^{-1}$ (5,0 ppm), que é o limite máximo permitido pela legislação brasileira. ${ }^{4}$ A mediana de concentração de cobre nessas amostras é de $5,49 \mathrm{mg}$ $\mathrm{L}^{-1}$, com variação entre $4,20 \times 10^{-2}$ a $19,9 \mathrm{mg} \mathrm{L}^{-1}$.

Não se observou, tanto para as amostras de aguardente de cana (Figura 2) como para as amostras de aguardente de mandioca (Material Suplementar, Figura 1S), qualquer correlação entre os teores de cobre e de CE.

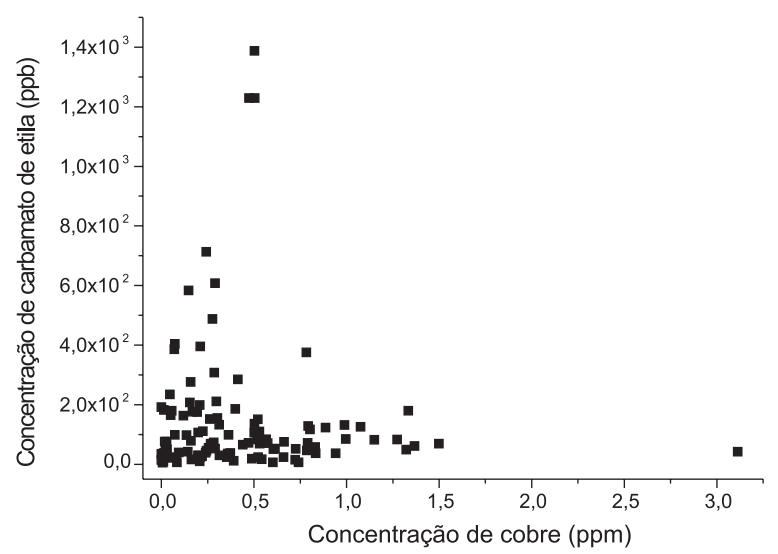

Figura 2. Concentração de carbamato de etila em $\mu g L^{-1}$ (ppb) versus concentração de cobre em $\mathrm{mg} \mathrm{L}^{-1}$ (ppm) para as 108 amostras de aguardente de cana

A radiação luminosa é outro fator que exerce influência na formação de CE. Conforme comentado anteriormente, a literatura indica que a incidência de luz entre 350 e $450 \mathrm{~nm}$ favorece a formação deste contaminante em uísques e "brandies" de frutas com caroço, matéria-prima que possui glicosídeos cianogênicos. ${ }^{30}$ Ainda segundo a literatura, ${ }^{15}$ o teor máximo de $\mathrm{CE}$ em uísque é atingindo depois de 3 dias de exposição dessa bebida à luz.

Amostras de tiquira acondicionadas em recipientes transparentes, quando expostos à luz difusa, apresentam aumento no teor de $\mathrm{CE}$. Possivelmente, as concentrações em níveis mais elevados de íons de cobre e cianeto na tiquira que na aguardente de cana ${ }^{21}$ sejam responsáveis pela variação observada. Este tema é permanente objeto de investigação em nosso Laboratório.

Nas mesmas 45 amostras de tiquira, foram determinadas as concentrações de íons cianeto (Tabela 3S). As concentrações dos íons cianeto apresentaram uma mediana de $8,00 \times 10^{-2} \mathrm{mg} \mathrm{L}^{-1}$ com intervalo de concentração entre $2,00 \times 10^{-3}$ a $1,33 \mathrm{mg} \mathrm{L}^{-1}$. As concentrações $^{21}$ de $\mathrm{CE}$ apresentaram um valor de mediana de $1,51 \times 10^{3}$ $\mu \mathrm{g} \mathrm{L}{ }^{-1}$ com intervalo de concentração entre 44,0 a $1,02 \times 10^{4} \mu \mathrm{g} \mathrm{L}^{-1}$. Esses resultados são compatíveis com os recentemente reportados na literatura $^{31}$ e de estudos não publicados. ${ }^{32}$

De forma semelhante ao discutido anteriormente em relação aos íons de cobre, também não ficou evidente uma correlação clara entre os teores de CE e os de íons cianeto (Material Suplementar, Figura 2S).

Provavelmente a ausência de correlação entre os teores de íons de cobre e CE na aguardente de cana e entre os teores de íons de cobre, CE e íons cianeto em aguardente de mandioca deve-se ao fato de que estas análises foram efetuadas em amostras já estabilizadas. Assim, há a necessidade de monitorar os teores destes precursores em amostras recém destiladas.

Foi constatada em nosso Laboratório a presença de uréia em amostras de aguardente de cana e de mandioca. Nas amostras de aguardente de cana, os teores de uréia são da ordem de $\mu \mathrm{g} \mathrm{L}^{-1}$, enquanto que nas aguardentes de mandioca estes teores são superiores, podendo atingir a faixa de $\mathrm{mg} \mathrm{L}^{-1}$. A presença de uréia nessas bebidas, mesmo após longos períodos de estocagem, sugere que a formação de $\mathrm{CE}$, através da reação de etanol com uréia, tanto em aguardente de cana como em tiquiras, se ocorrer, deve ser muito lenta.

\section{CONCLUSÕES}

Os resultados coletados para carbamato de etila nas 380 amostras de aguardente (Material Suplementar, Tabela 4S) indicam que 50\% destas atendem os requisitos previstos na legislação brasileira com respeito aos teores de CE. Em relação aos teores de cobre, num total de 294 amostras de aguardente, $70 \%$ atendem os limites previstos na legislação brasileira. Indicam ainda que os teores de CE em aguardente de cana comercial diminuíram consideravelmente ao longo dos últimos anos, sugerindo que a inclusão de seu controle na legislação brasileira não irá de forma alguma inibir o setor produtivo. Pelo contrário, percebe-se uma preocupação cada vez maior dos produtores em melhorar a qualidade da aguardente visando provavelmente produzir uma bebida tipo exportação e se adequar à legislação brasileira. ${ }^{4}$

Conforme citado anteriormente, ${ }^{19}$ confirmou-se por meio de resultados obtidos para um universo maior de amostras e ao longo de 5 anos que as cachaças "artesanais" (alambique) tendem a apresentar um teor de CE inferior ao das cachaças ditas "industriais" (coluna). A separação do destilado artesanal nas frações cabeça, coração e cauda aliada ao fato do CE ser mais abundante na fração cabeça é uma possível explicação para tal observação.

Verificou-se que os teores de CE em amostras de aguardente de cana são estáveis por um período de pelo menos 3 anos e que sua formação deve se completar logo após a produção do destilado. Não foi possível até o momento estabelecer de forma definitiva qualquer correlação entre a coloração do recipiente e o teor de CE em amostras comerciais, nem tampouco correlacionar a presença de CE com as concentrações dos precursores, íons de cobre e cianeto em tiquira. Tal fato deve-se provavelmente ao tempo transcorrido entre a produção das aguardentes e a análise do CE e de seus precursores.

\section{MATERIAL SUPLEMENTAR}

Está disponível em http://quimicanova.sbq.org.br, na forma de arquivo PDF, com acesso livre.

\section{AGRADECIMENTOS}

À CAPES, ao CNPq e à FAPESP (processo no 2001/12934 e 2004/15985-3) pelo apoio financeiro.

\section{REFERÊNCIAS}

1. Zimmerli, B.; Schlatter, J.; Mutat. Res. 1991, 259, 325.

2. Galinaro, C. A.; Cardoso, D. R.; Franco, D. W.; J. Agric. Food Chem. 2007, 55, 3141.

3. Rodrigues Filho, M. G.; Leite-Neto, A. F.; Aquino, F. W. B.; Plepis, A. M.; Rodrigues-Filho, U. F.; Franco, D. W.; Quim. Nova 2007, 30, 1115 .

4. BRASIL. Instrução Normativa n $^{\circ} 13$, de 29 de junho de 2005, Ministério da Agricultura Pecuária e Abastecimento; Diário Oficial da União, seção 1, p. 3-4, de 30/06/2005.

5. Ought, C. S.; J. Agric. Food Chem. 1976, 24, 323.

6. http://www.cfsan.fda.gov/ frf/ecaction.html, acessada em Fevereiro 2008.

7. Hasnip, S.; Caputi, A.; Crews, C.; Brereton, P.; Food Addit. Contam. 2004, 21, 1155

8. Monteiro, F. F.; Bisson, L. F.; Am. J. Enology and Viticulture 1992, 43, 18. 
9. Ough, C. S.; Huang, Z.; An, D.; Stevens, D.; Am. J. Enology and Viticulture 1991, 42, 26

10. Stevens, D. F.; Ough, C. S.; Am. J. Enology and Viticulture 1993, 44, 309.

11. Tegmo-Larsson, I. M.; Henick-Kling, T.; Am. J. Enology and Viticulture 1990, 41, 269.

12. Solymosy, F.; Antoni, F.; Fedorcsák, I.; J. Agric. Food Chem. 1978, 26 , 500.

13. Aresta, M.; Boscolo, M.; Franco, D. W.; J. Agric. Food Chem. 2001, 49, 2819.

14. Swanston, J. S.; J. Sci. Food Agric. 1999, 79, 745

15. Mackenzie, W. M.; Clyne, A. H.; Macdonald, L. S.; J. Institute of Brewing 1990, 96, 223.

16. Cook, R.; McCaig, N.; McMillan, J. M. B.; Lumsden, W. B.; J. Institute of Brewing 1990, 96, 233.

17. Aylott, R. I.; Cochrane, G. C.; Leonard, M. J.; Macdonald, L. S.; Mackenzie, W. M.; Mcneish, A. S.; Walker, D. A.; J. Institute of Brewing 1990, 96, 213

18. McGill, D. J.; Morley, A. S.; J. Institute of Brewing 1990, 96, 245.

19. Andrade-Sobrinho, L. G.; Boscolo, M.; Lima-Neto, B. S.; Franco, D. W.; Quim. Nova 2002, 25, 1074.

20. Boscolo, M.; Tese de Doutorado, Universidade de São Paulo, Brasil, 2001.
21. Andrade-Sobrinho, L. G.; Tese de Doutorado, Universidade de São Paulo, Brasil, 2004

22. Cappelini, L. T. D.; Silva, A. A.; Buchviser, S. F.; Andrade-Sobrinho, L. G.; Franco, D. W.; Engarrafador Moderno 2007, 153, 34.

23. Nascimento, R. F.; Bezerra, C. W.; Furuya, S. M. B.; Schultz, M. S.; Polastro, L. R.; Lima-Neto, B. S.; Franco, D. W.; J. Food Comp. Anal. 1999, 17, 17.

24. Lambert, J. L.; Ramasamy, J.; Paukstelis, J. V.; Anal. Chem. 1975, 47, 916

25. Lima, U. A.; Aguardente: fabricação em pequenas destilarias, Ed. FEALQ: Piracicaba, 1999.

26. Bruno, S. N. F.; Vaitsman, D. S.; Kunigami, C. N.; Brasil, M. G.; Food Chem. 2007, 104, 1345.

27. Macedo, M. J., comunicação pessoal.

28. Mutton, M. J. R.; Mutton, M. A. Em Tecnologia de Bebidas; VenturiniFilho, W. G., ed.; Edgard Blücher: São Paulo, 2005; cap. 20.

29. http://sigma-aldrich.com, acessada em Janeiro 2008.

30. Guerain, J.; Leblond, N. Em Elaboration et connaissance des spititeux; Cantagrel, R., ed.; Tec \& Doc: Paris, 1998, p. 330.

31. Furtado, J. L. B.; Bezerra, C. W. B.; Marques, E. P.; Marques, A. L. B.; Ciênc. Tecnol. Aliment. 2007, 27, 694.

32. Tubino, M., comunicação pessoal. 
Luiz Gualberto de Andrade Sobrinho, Luciana Tereza Dias Cappelini, Alexandre Ataíde da Silva, Carlos Alexandre Galinaro, Silmara França Buchviser, Daniel Rodrigues Cardoso e Douglas Wagner Franco*

Instituto de Química de São Carlos, Universidade de São Paulo, CP 780, 13560-970 São Carlos - SP, Brasil

Tabela 1S. Concentração de carbamato de etila (CE) em amostras de aguardente comercial ( $\mu$ g $\left.\mathrm{L}^{-1}\right)$ após 36 meses de repouso e abrigo da luz

\begin{tabular}{lccc}
\hline Amostra & Resultados obtidos em 2001 & Resultados obtidos em 2004 & Diferenças entre os valores (\%) \\
\hline 1 & 902 & 875 & $-3,0$ \\
2 & 50,0 & 53,0 & 6,0 \\
3 & 313 & 318 & 1,6 \\
4 & 357 & 338 & $-5,3$ \\
5 & $1,68 \times 10^{3}$ & $1,75 \times 10^{3}$ & 4,2 \\
6 & 266 & 258 & $-3,0$ \\
7 & 613 & 614 & 0,16 \\
8 & $2,61 \times 10^{3}$ & 0,34 \\
9 & 507 & $2,62 \times 10^{3}$ & 6,7 \\
10 & 705 & 541 & 1,9 \\
11 & 680 & 719 & $-2,8$ \\
13 & 484 & 661 & $-3,9$ \\
15 & $3,48 \times 10^{3}$ & 465 & 6,6 \\
\hline
\end{tabular}

*e-mail: douglas@iqsc.usp.br 
Tabela 2S. Valores de teor alcoólico (\% v/v), concentração de carbamato de etila (CE, $\left.\mu \mathrm{g} \mathrm{L} \mathrm{L}^{-1}\right)$ e de cobre $\left(\mathrm{mg} \mathrm{L}^{-1}\right)$ em 108 amostras de aguardente de cana referentes ao Projeto de Tipificação da Aguardente do Estado de São Paulo

\begin{tabular}{|c|c|c|c|c|c|c|c|c|c|c|c|}
\hline Amostra & $\begin{array}{c}\text { Teor } \\
\text { alcoólico }\end{array}$ & $\mathrm{CE}^{*}$ & Cobre* & Amostra & $\begin{array}{c}\text { Teor } \\
\text { alcoólico }\end{array}$ & $\mathrm{CE}^{*}$ & Cobre* & Amostra & $\begin{array}{c}\text { Teor } \\
\text { alcoólico }\end{array}$ & $\mathrm{CE}^{*}$ & Cobre* \\
\hline 1 & 41,4 & $1,23 \times 10^{3}$ & 0,504 & 19 & 49,5 & 199 & 0,207 & 37 & 45,5 & $<$ L.Q. & 0,209 \\
\hline 2 & 40,1 & 76,0 & $2,80 \times 10^{-2}$ & 20 & 54,9 & 713 & 0,242 & 38 & 46,8 & <L.Q. & 0,162 \\
\hline 3 & 46,1 & 99,0 & 0,363 & 21 & 40,5 & 85,0 & 0,996 & 39 & 68,5 & 55,0 & 0,255 \\
\hline 4 & 41,3 & 175 & 0,193 & 22 & 74,3 & 404 & $7,20 \times 10^{-2}$ & 40 & 55,4 & $<$ L.Q. & 0,220 \\
\hline 5 & 49,8 & 488 & 0,275 & 23 & 56,5 & 74,0 & 0,282 & 41 & 48,3 & 71,0 & 0,574 \\
\hline 6 & 45,2 & 151 & 0,521 & 24 & 50,7 & 98,0 & 0,135 & 42 & 40,8 & 75,0 & 0,662 \\
\hline 7 & 49,4 & 208 & 0,154 & 25 & 53,0 & 72,0 & 0,789 & 43 & 25,0 & 61,0 & 1,37 \\
\hline 8 & 49,3 & 308 & 0,285 & 26 & 45,4 & 42,0 & 0,142 & 44 & 49,9 & 53,0 & 0,289 \\
\hline 9 & 59,7 & $1,39 \times 10^{3}$ & 0,503 & 27 & 40,3 & 123 & 0,887 & 45 & 56,6 & 47,0 & 0,786 \\
\hline 10 & 49,3 & 69,0 & 0,270 & 28 & 52,7 & 79,0 & 0,159 & 46 & 41,1 & <L.Q. & 0,833 \\
\hline 11 & 53,0 & 136 & 0,502 & 29 & 45,8 & 66,0 & 0,440 & 47 & 49,4 & $<$ L.Q. & 0,723 \\
\hline 12 & 21,1 & 180 & 1,33 & 30 & 39,2 & 126 & 1,07 & 48 & 50,0 & 46,0 & 0,243 \\
\hline 13 & 79,6 & <L.Q. & $1,40 \times 10^{-2}$ & 31 & 45,4 & 176 & 0,167 & 49 & 44,6 & 51,0 & 0,605 \\
\hline 14 & 25,1 & 106 & 0,501 & 32 & 55,2 & 152 & 0,262 & 50 & 45,7 & <L.Q. & 0,541 \\
\hline 15 & 49,1 & 155 & 0,302 & 33 & 40,5 & 18,7 & 0,488 & 51 & 32,2 & $1,23 \times 10^{3}$ & 0,473 \\
\hline 16 & 26,0 & 117 & 0,803 & 34 & 52,2 & 58,0 & 0,830 & 52 & 38,5 & 49,0 & 1,32 \\
\hline 17 & 47,0 & 396 & 0,210 & 35 & 38,2 & <L.Q. & 0,199 & 53 & 47,3 & $<$ L.Q. & 0,659 \\
\hline 18 & 52,1 & 584 & 0,146 & 36 & 58,6 & <L.Q. & 0,565 & 54 & 33,0 & 69,0 & 0,530 \\
\hline 55 & 38,6 & <L.Q. & 0,197 & 73 & 50,1 & 83,0 & 0,516 & 91 & 38,0 & 50,0 & $3,00 \times 10^{-2}$ \\
\hline 56 & 52,3 & $<$ L.Q. & $1,00 \times 10^{-2}$ & 74 & 54,4 & 182 & $1,00 \times 10^{-2}$ & 92 & 42,3 & 100 & $7,20 \times 10^{-2}$ \\
\hline 57 & 48,7 & 211 & 0,296 & 75 & 65,4 & 76,0 & $1,00 \times 10^{-2}$ & 93 & 42,4 & < L.Q. & 0,358 \\
\hline 58 & 54,1 & 386 & $7,00 \times 10^{-2}$ & 76 & 50,0 & 235 & $4,60 \times 10^{-2}$ & 94 & 49,5 & < L.Q. & 0,783 \\
\hline 59 & 48,1 & 276 & 0,158 & 77 & 48,6 & 180 & $5,50 \times 10^{-2}$ & 95 & 53,7 & 608 & 0,290 \\
\hline 60 & 38,5 & $<$ L.Q. & 0,207 & 78 & 48,7 & 133 & 0,312 & 96 & 41,4 & 285 & 0,413 \\
\hline 61 & 65,3 & $<$ L.Q. & $1,00 \times 10^{-2}$ & 79 & 46,3 & 82,0 & 1,15 & 97 & 49,2 & < L.Q. & 0,239 \\
\hline 62 & 40,0 & $<$ L.Q. & $1,00 \times 10^{-2}$ & 80 & 62,2 & $<$ L.Q. & 0,391 & 98 & 55,2 & 186 & 0,399 \\
\hline 63 & 51,4 & $<$ L.Q. & 0,740 & 81 & 63,0 & $<$ L.Q. & 0,314 & 99 & 51,8 & $<$ L.Q. & 0,198 \\
\hline 64 & 53,4 & < L.Q. & $1,00 \times 10^{-2}$ & 82 & 56,7 & <L.Q. & $9,40 \times 10^{-2}$ & 100 & 56,6 & 128 & 0,793 \\
\hline 65 & 51,2 & 163 & 0,119 & 83 & 48,7 & $<$ L.Q. & $7,80 \times 10^{-2}$ & 101 & 54,9 & $<$ L.Q. & 0,602 \\
\hline 66 & 54,6 & 111 & 0,223 & 84 & 50,9 & <L.Q. & 0,941 & 102 & 53,7 & 35,0 & $1,00 \times 10^{-2}$ \\
\hline 67 & 38,1 & 110 & 0,529 & 85 & 54,4 & < L.Q. & $8,40 \times 10^{-2}$ & 103 & 53,6 & 192 & $1,00 \times 10^{-2}$ \\
\hline 68 & 46,4 & $<$ L.Q. & 0,352 & 86 & 72,5 & <L.Q. & $5,20 \times 10^{-2}$ & 104 & 46,6 & $<$ L.Q. & 0,522 \\
\hline 69 & 52,6 & 72,0 & 0,470 & 87 & 40,4 & 132 & 0,988 & 105 & 39,9 & $<$ L.Q. & 0,372 \\
\hline 70 & 44,5 & 68,0 & 0,265 & 88 & 44,0 & 67,0 & 0,725 & 106 & 46,1 & 165 & $5,00 \times 10^{-2}$ \\
\hline 71 & 54,6 & 83,0 & 1,27 & 89 & 42,8 & 69,0 & 0,613 & 107 & 49,5 & 107 & 0,199 \\
\hline 72 & 51,4 & 52,0 & $1,00 \times 10^{-2}$ & 90 & 36,4 & 42,0 & 3,11 & 108 & 44,1 & 69,0 & 1,49 \\
\hline
\end{tabular}

* Os valores de concentrações de carbamato de etila (CE) e cobre foram corrigidos utilizando um valor de grau alcoólico de $38 \%$ v/v. $<$ L.Q. = menor que o limite de quantificação. 
Tabela 3S. Valores de concentrações de carbamato de etila $\left(\mathrm{CE}, \mu \mathrm{g} \mathrm{L} \mathrm{L}^{-1}\right)$, de cobre $\left(\mathrm{mg} \mathrm{L}^{-1}\right)$ e cianeto $\left(\mathrm{mg} \mathrm{L}^{-1}\right)$, em 45 amostras de aguardente de mandioca (tiquira)

\begin{tabular}{|c|c|c|c|c|c|c|c|}
\hline Amostra & $\mathrm{CE}$ & Cobre & Cianeto & Amostra & $\mathrm{CE}$ & Cobre & Cianeto \\
\hline 1 & $1,02 \times 10^{4}$ & 2,44 & $8,00 \times 10^{-3}$ & 24 & $2,41 \times 10^{3}$ & 6,47 & 0,123 \\
\hline 2 & $1,25 \times 10^{3}$ & 2,90 & $2,20 \times 10^{-2}$ & 25 & 206 & $9,60 \times 10^{-2}$ & $5,00 \times 10^{-3}$ \\
\hline 3 & $1,04 \times 10^{3}$ & 8,52 & $8,00 \times 10^{-2}$ & 26 & 197 & $6,00 \times 10^{-2}$ & $2,00 \times 10^{-3}$ \\
\hline 4 & $5,20 \times 10^{3}$ & 4,01 & $9,50 \times 10^{-2}$ & 27 & 198 & $4,20 \times 10^{-2}$ & $2,00 \times 10^{-3}$ \\
\hline 5 & $3,87 \times 10^{3}$ & 4,98 & $2,10 \times 10^{-2}$ & 28 & 193 & $4,50 \times 10^{-2}$ & $2,00 \times 10^{-3}$ \\
\hline 6 & 837 & 7,63 & $7,00 \times 10^{-2}$ & 29 & $1,79 \times 10^{3}$ & 3,66 & $1,40 \times 10^{-2}$ \\
\hline 7 & $2,69 \times 10^{3}$ & 3,87 & $2,20 \times 10^{-2}$ & 30 & 198 & $7,20 \times 10^{-2}$ & $5,00 \times 10^{-3}$ \\
\hline 8 & $3,13 \times 10^{3}$ & 3,77 & $8,50 \times 10^{-2}$ & 31 & 193 & $9,20 \times 10^{-2}$ & $9,00 \times 10^{-3}$ \\
\hline 9 & $3,39 \times 10^{3}$ & 4,62 & $3,80 \times 10^{-2}$ & 32 & $3,11 \times 10^{3}$ & 6,46 & 0,135 \\
\hline 10 & $6,10 \times 10^{3}$ & 6,49 & $9,20 \times 10^{-2}$ & 33 & 44,0 & 8,95 & 0,180 \\
\hline 11 & $3,80 \times 10^{3}$ & 6,50 & 0,110 & 34 & $1,72 \times 10^{3}$ & $6,10 \times 10^{-2}$ & $3,20 \times 10^{-2}$ \\
\hline 12 & 651 & 6,47 & 0,123 & 35 & $1,25 \times 10^{3}$ & 19,9 & 0,169 \\
\hline 13 & $5,63 \times 10^{3}$ & 2,44 & $8,00 \times 10^{-3}$ & 36 & $1,08 \times 10^{3}$ & 17,2 & 0,231 \\
\hline 14 & $1,34 \times 10^{3}$ & 2,90 & $2,20 \times 10^{-2}$ & 37 & $1,51 \times 10^{3}$ & 7,54 & 0,239 \\
\hline 15 & $2,89 \times 10^{3}$ & 8,52 & $8,00 \times 10^{-2}$ & 38 & $2,02 \times 10^{3}$ & 7,71 & 0,377 \\
\hline 16 & $1,67 \times 10^{3}$ & 4,01 & $9,50 \times 10^{-2}$ & 39 & $2,11 \times 10^{3}$ & 12,3 & $9,10 \times 10^{-2}$ \\
\hline 17 & $1,90 \times 10^{3}$ & 4,98 & $2,10 \times 10^{-2}$ & 40 & 742 & 19,8 & $9,70 \times 10^{-2}$ \\
\hline 18 & $2,45 \times 10^{3}$ & 7,63 & $7,00 \times 10^{-2}$ & 41 & 345 & 5,49 & $6,00 \times 10^{-2}$ \\
\hline 19 & $1,04 \times 10^{3}$ & 3,87 & $2,20 \times 10^{-2}$ & 42 & 325 & 8,88 & 1,33 \\
\hline 20 & $1,25 \times 10^{3}$ & 3,77 & $8,50 \times 10^{-2}$ & 43 & 379 & 6,37 & $7,10 \times 10^{-2}$ \\
\hline 21 & $6,13 \times 10^{3}$ & 4,62 & $3,80 \times 10^{-2}$ & 44 & 729 & 10,1 & 0,204 \\
\hline 22 & $2,76 \times 10^{3}$ & 6,49 & $9,20 \times 10^{-2}$ & 45 & 440 & 9,03 & 0,231 \\
\hline 23 & $2,29 \times 10^{3}$ & 6,50 & 0,110 & & & & \\
\hline
\end{tabular}

Tabela 4S. Mediana da concentração $\left(\mu \mathrm{g} \mathrm{L}^{-1}\right)$ de carbamato de etila (CE) em amostras analisadas pelo LDQA entre os anos 2001 a 2006

\begin{tabular}{|c|c|c|c|c|c|c|c|c|}
\hline Amostras & $\begin{array}{c}\mathrm{N}^{\circ} \text { de amostras } \\
\text { de } \mathrm{CE}\end{array}$ & $\begin{array}{c}\text { Mediana } \\
{[\mathrm{CE}]\left(\mu \mathrm{g} \mathrm{L}^{-1}\right)}\end{array}$ & $\begin{array}{c}\text { Máximo } \\
{[\mathrm{CE}]\left(\mu \mathrm{g} \mathrm{L}^{-1}\right)}\end{array}$ & $\begin{array}{c}\% \text { de amostras } \\
\text { com }[\mathrm{CE}] \\
<150 \mu \mathrm{g} \mathrm{L}^{-1}\end{array}$ & $\begin{array}{c}\mathrm{N}^{\circ} \text { de amostras } \\
\text { de } \mathrm{Cu}\end{array}$ & $\begin{array}{c}\text { Mediana } \\
{[\mathrm{Cu}]\left(\mathrm{mg} \mathrm{L}^{-1}\right)}\end{array}$ & $\begin{array}{c}\text { Máximo } \\
{[\mathrm{Cu}]\left(\mathrm{mg} \mathrm{L}^{-1}\right)}\end{array}$ & $\begin{array}{c}\% \text { de amostras } \\
\text { com }[\mathrm{Cu}] \\
<5,0 \mathrm{mg} \mathrm{L}^{-1}\end{array}$ \\
\hline $\begin{array}{l}\text { Comerciais } \\
1993\end{array}$ & - & - & - & - & 74 & 3,18 & 14,3 & 72 \\
\hline $\begin{array}{l}\text { Comerciais } \\
2001\end{array}$ & 126 & 479 & $5,69 \times 10^{3}$ & 21 & - & - & - & - \\
\hline $\begin{array}{l}\text { Tipificação } \\
2002\end{array}$ & 108 & 107 & $1,39 \times 10^{3}$ & 72 & 108 & 3,93 & 29,8 & 60 \\
\hline $\begin{array}{l}\text { V BMCFB } \\
2004\end{array}$ & 36 & 108 & 460 & 67 & 36 & 1,50 & 11,4 & 81 \\
\hline $\begin{array}{l}\text { Comerciais } \\
2005\end{array}$ & 41 & 163 & $1,16 \times 10^{3}$ & 42 & 7 & 3,81 & 5,91 & 57 \\
\hline $\begin{array}{l}\text { VI BMCFB } \\
2006\end{array}$ & 34 & 85,0 & 646 & 76 & 34 & 1,26 & 12,5 & 82 \\
\hline $\begin{array}{l}\text { Comerciais } \\
2006\end{array}$ & 35 & 138 & $1,67 \times 10^{3}$ & 51 & 35 & 3,27 & 14,6 & 74 \\
\hline Total & 380 & & & & 294 & & & \\
\hline
\end{tabular}

$[\mathrm{CE}]=$ concentração de carbamato de etila. $[\mathrm{Cu}]=$ concentração de cobre 


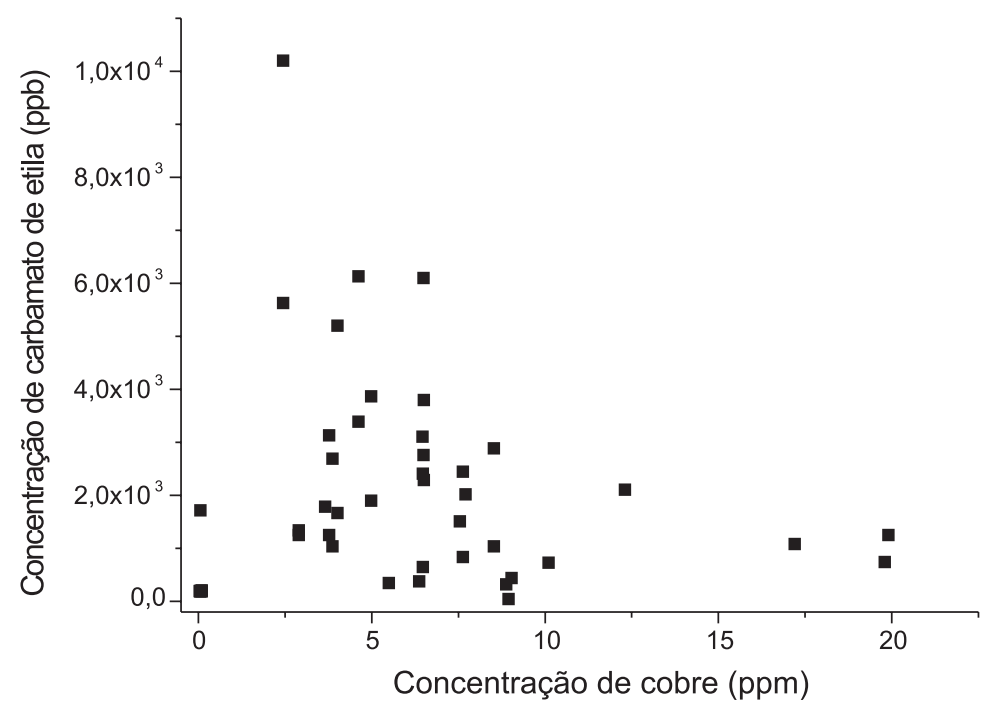

Figura 1S. Concentração de carbamato de etila em $\mu \mathrm{g} L^{-1}(\mathrm{ppb})$ versus concentração de cobre em $m g L^{-1}$ (ppm) em 45 amostras de aguardente de mandioca (tiquira)

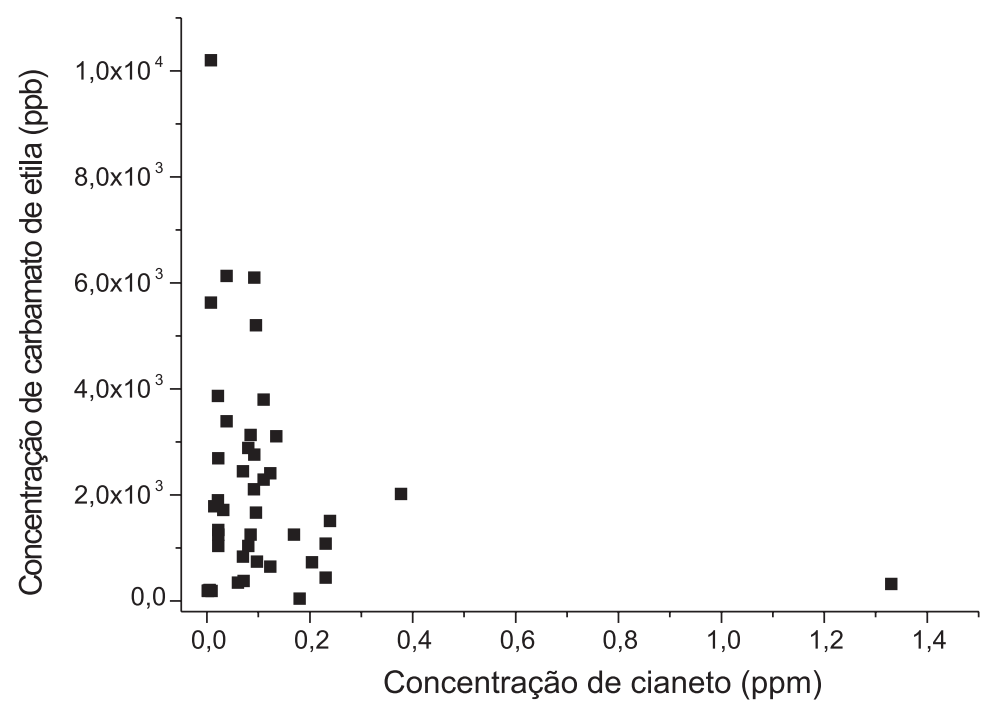

Figura 2 S. Concentração de carbamato de etila em $\mu g L^{-1}(p p b)$ versus concentração de cianeto em $m g L^{-1}$ (ppm) em 45 amostras de aguardente de mandioca (tiquira) 